\title{
Does simulated microgravity induce significant changes in human gut microbiota? New answers with dry immersion, an innovative ground-based model.
}

\section{Maxence Jollet}

Universite de Montpellier https://orcid.org/0000-0003-1191-4791

\section{Bénédicte Goustard}

INRA Centre de Montpellier

Mahendra Mariadassou

INRA Centre de Jouy-en-Josas

Olivier Rué

INRA Centre de Jouy-en-Josas

Vincent Ollendorff

INRA Centre de Montpellier

\section{Barbara Vernus}

INRA Centre de Montpellier

\section{Anne Bonnieu}

INRA Centre de Montpellier

\section{Kévin Nay}

Université de Rennes/ENS

Marie-Pierre Bareilles

Institut de Médecine et Physiologie Spatiales

\section{Arnaud Beck}

Institut de Médecine et de Physiologie Spatiales

Guillemette Gauquelin-Koch

CNES

Frédéric Derbré

Université de Rennes/ENS

Christelle Koechlin-Ramonatxo ( $\nabla$ christelle.ramonatxo@umontpellier.fr ) Universite de Montpellier https://orcid.org/0000-0001-6112-0864 
Keywords: Hypoactivity, commensal bacteria, intestine, flora, organ cross-talk, muscle atrophy.

Posted Date: August 10th, 2020

DOI: https://doi.org/10.21203/rs.3.rs-52630/v1

License: (c) (1) This work is licensed under a Creative Commons Attribution 4.0 International License. Read Full License 


\section{Abstract}

Background A new problematic on the gut microbiota of the astronauts and the effects of microgravity emerged recently as that bacteria community is sensitive to physical (in)activity which could be hampered during spaceflights. Therefore, the objective of our study was thus to determine the effects of dry immersion, an innovative ground-based human model of simulated microgravity, on human gut microbiota composition. We collected stools from 14 healthy men before and after 5 days of Dry Immersion to determine taxonomic profiles by $16 \mathrm{~S}$ metagenomic.

Results Our analyses show preservation of a-diversity through Observed, Chao1, Shannon and InvSimpson indices. $\beta$-diversity is also not impacted by Dry Immersion as represented by PCoA plots with Jaccard, Bray-Curtis and UniFrac indices. Phyla abundances for OTUs associated to Bacteroidetes ${ }^{P}$, Firmicutes $^{P}$, Proteobacteria $^{P}$ and Actinobacteria $^{P}$ are also preserved. Interestingly, metagenomics analysis of the 32 families and 44 associated genera underscored that OTUs associated to Clostridiales ${ }^{O}$ order and Lachnospiraceae ${ }^{F}$ family are increased $(p<0.01)$ belonging to Firmicutes ${ }^{P}$ phylum.

Conclusion The diversity and global composition of the gut microbiome remained unaltered in response to Dry Immersion confirming the robustness of gut microbiota. However, it's sufficient to led to several significant changes at the lower taxonomy levels. This suggests that the human gut microbiota, with its known strong impact on human health and performance, is a potential biological target of microgravity and underscores the need to investigate further this new field of research on gut microbiota microgravity.

Trial registration: ClinicalTrials.gov Identifier NCT03915457- Registered 16 April 2019 - Retrospectively registered - https://clinicaltrials.gov/ct2/show/NCT03915457.

\section{Background}

Numerous notable human physiological changes occur during spaceflights due to specific, extreme environmental conditions (microgravity, increased radiation, isolation, anxiety, sleep deprivation...) that can adversely affect crew health and productivity [1]. For example, the prolonged exposure to weightlessness induces major problems of bone loss, cardiovascular deconditioning, muscle weakening, sensorimotor and neurovestibular disturbances, as well as regulatory and immune disorders [1]. To identify, prevent and mitigate these significant changes poses very inspiring and demanding challenges for future deep space exploration missions associated with increased duration of mission.

Recent research has revealed that the gut microbiota impacts the host physiology and human health [2]. Indeed, the data obtained on earth argue to preserve an optimized gut microbiota composition, as its disruption/deregulation of these bacteria communities referred to as dysbiosis, is associated with health disorders [3] and/or altered physical performance [4-8]. As a modulator of the immune system, the gut microbiota contributes to intestinal homeostasis and integrity (permeability and inflammation) and exerts 
a nutritional role via its bacteria-derived metabolites [4]. The microbiota in our gut performs many essential functions including vitamin production, regulation of lipid metabolism and state-grade inflammation, as well as short chain fatty acid production as fuel for epithelial cells and regulation of gene expression [5]. Moreover, inter-organ cross talks between gut microbiota and other organs of the host, such as brain or adipose tissue have been identified [2]. Interestingly, our laboratory revealed a functional cross-talk in mice between gut microbiota and skeletal muscle. Skeletal muscles are essential tissues involved in energy balance regulation, body weight composition and physical performance and are altered in astronauts during spaceflights. We indeed showed in mice that the dysbiosis induced by a large spectrum antibiotic treatment during 21 days decreased running endurance and exacerbated muscle fatigue, as the extensor digitorum longus muscle fatigue index in an ex vivo contractile test was reduced. Importantly, the muscle endurance capacity was efficiently normalized by natural reseeding [6]. This result was further supported by a recent Swedish study using the germ free (GF) model and showing that the gut microbiota contributes to skeletal muscle mass and function in mice [7]. Indeed, GF mice were shown to display reduced muscle mass and signs of muscle atrophy indicated by an increased activation of AMPK-Fox03-Atrogin cascade simultaneously with a decreased hindlimb grip strength [7]. Treating GF mice with short-chain fatty acids partly reversed the skeletal muscle loss of function [7].

The analysis of the literature highlights that these rich and diverse bacteria communities, including thousands of different species, are modulated by some of the key spaceflight constraints: disruption of nutritional balance, potential use of antibiotics to mitigate the "compromised adaptative immune function" associated with spaceflights, and also the microgravity [1]. A number of studies have thus investigated how spaceflight affects the microbial composition of the astronauts' gastrointestinal tract. Compositional changes in cultivable bacteria collected from the gastrointestinal tract of astronauts spending up to 2 months in space were detected [1] with post-flight reduction of Lactobacilli ${ }^{G}$ genus, and increased levels of Enterobacteria ${ }^{F}$ and Clostridia $^{F}$ families. However, these studies were only based on cultivable bacteria and not on the taxonomic profiles. Two recent NASA studies should also be highlighted: the NASA twin project, which compared one twin astronaut microbiota before, during, and after a 1-year mission on-board the International Space Station (ISS); his twin serving as a genetically matched ground control [9], and the Astronaut's Microbiome (AM) project which investigated the impact of the spaceflight and the confined environment on crewmembers spending 6 months to 1 year on-board the ISS [10]. In both studies, gastrointestinal microbiota alterations and microbiome changes were observed. If the number of species remained unchanged, the analysis of the AM project identified 17 gastrointestinal genera OTUs whose their abundances were significantly changed in space, mostly belonging to the Phylum Firmicutes ${ }^{P}$ and to the Order Clostridiales $^{O}$. All these studies are undergoned on very few human cases limiting statistic power and need to be confirmed with complementary earthcontrolled study including larger population. Indeed, the effects of microgravity on the gut microbiota composition remain poorly understood in humans despite its major role in the host' health and performance. In this context, ground based human models appeared pertinent to characterize the adaptation of human gut microbiota to microgravity effects. 
Dry Immersion (DI) is an innovative approach that accurately reproduces the effects of microgravity more quickly than the head-down bedrest model. It consists of immersing an individual in thermally neutral water and covered with an elastic waterproof tarpaulin. The subject therefore lies freely lying in the water while remaining dry, hence the term dry immersion [11]. Unlike the bedrest, this model completely limits the possibility for the subject to rest on supports. Recent studies on cardiovascular, postural and neuromuscular changes and comparisons of the effects of 21 days of bedrest and 3 days of Dry Immersion have shown similar effects in their amplitude, suggesting that the immersion induces physiological changes in the weightlessness much faster [12]. This allows us to be as close as possible to the modalities of muscular inactivity encountered in real situations in space compared to the classic bed rest model [13].

Therefore, the purpose of the present study is to identify in healthy humans the effects of simulated microgravity and extreme physical inactivity on $a$ and $\beta$-diversity analysis of taxonomic profiles and differential abundance analysis of phyla, order and families with 16S rRNA gene metagenomics analysis. For this purpose, we used the Dry Immersion model that accurately reproduces the effects of microgravity, and exposed healthy young volunteers for 5 days to this innovative ground-based human model. In addition, a group of participants underwent venoconstrictive thigh cuffs protocol, a countermeasure to microgravity used to sequester fluids in the lower limbs.

\section{Methods}

\section{Participants}

20 healthy men were recruited to be exposed to 5 days of Dry Immersion. Two subjects were excluded before BDC-5 ( 5 days Before Data Collection) for reasons unrelated to protocol. A total of eighteen subjects were included in the study and randomly divided at BDC-2 into Control or Cuffs group (9/9 split). Participants were anonymized and designated in data sets by single letters. Subjects $B, E, F, I, K, M, O, Q$ and $S$ were included in control group while A, C, D, G, H, J, N, P and R were included in Cuffs group. All subjects were informed about the experimental procedures and gave their written consent. The experimental protocol conformed to the standards set by the Declaration of Helsinki and was approved by the local Ethic Committee (CPP Est III: October 2, 2018, $n^{\circ}$ ID RCB 2018-A01470-55) and French Health Authorities (ANSM: August 13, 2018, ClinicalTrials.gov Identifier: NCT03915457). Baseline group characteristics are detailed in Table 1. There was no significant difference between groups at baseline (Table 1). 
Table 1

Baseline group characteristics at BDC-2. Data are the mean \pm SD. Statistical significance was checked using an unpaired T-test. BDC $=$ Baseline Data Collection; $\mathrm{BMI}=$ Body Mass Index; $\mathrm{VO}_{2} \mathrm{max}=$ Maximal $\mathrm{O}_{2}$ Consumption; $\mathrm{HR}=$ Heart rate; $\mathrm{T}=$ Temperature; $\mathrm{SPB}=$ Systolic $\mathrm{Blood}$ Pressure; $\mathrm{DPB}=$ Diastolic Blood Pressure; $\mathrm{DI}=$ Dry Immersion; $\mathrm{CTL}=$ control group (no thigh cuffs); CUFF = cuffs group (with thigh cuffs);

$A L L=$ all subjects.

\begin{tabular}{|c|c|c|c|}
\hline & $\operatorname{CTL}(n=9)$ & CUFF $(n=9)$ & ALL $(n=18)$ \\
\hline Age (years) & $33.4 \pm 7.1$ & $33.8 \pm 3.7$ & $33.6 \pm 5.5$ \\
\hline Height (cm) & $176 \pm 6$ & $180 \pm 4$ & $178 \pm 6$ \\
\hline Weight (kg) & $73.9 \pm 7.5$ & $74.3 \pm 8.8$ & $74.4 \pm 8.0$ \\
\hline BMI $\left(k g / m^{2}\right)$ & $23.9 \pm 1.7$ & $22.7 \pm 1.8$ & $23.5 \pm 1.9$ \\
\hline $\mathrm{VO}_{2} \max (\mathrm{ml} / \mathrm{min} / \mathrm{kg})$ & $46.5 \pm 8.1$ & $46.9 \pm 5.8$ & $46.7 \pm 6.9$ \\
\hline Morning HR (bpm) & $57 \pm 6$ & $58 \pm 8$ & $58 \pm 7$ \\
\hline Morning $\mathrm{T}\left({ }^{\circ} \mathrm{C}\right)$ & $36.4 \pm 0.3$ & $36.4 \pm 0.5$ & $36.4 \pm 0.4$ \\
\hline Morning SBP $(\mathrm{mmHg})$ & $115 \pm 11$ & $117 \pm 10$ & $116 \pm 10$ \\
\hline Morning DBP (mmHg) & $68 \pm 5$ & $68 \pm 9$ & $68 \pm 7$ \\
\hline
\end{tabular}

The study was conducted at the MEDES space clinic, Toulouse, France from 19/11/2018 to 23/03/2019. Subjects arrived in the evening of BDC-5 and left in the morning of R+2 (Recovery day 3 ). The experimental protocol included four days of ambulatory baseline measurements before immersion (BDC4 to BDC-1), five days (120 hours) of Dry Immersion (DI1 to DI5) and two days of ambulatory recovery $(\mathrm{RO}, \mathrm{R}+1)$. In addition, a week prior to beginning the protocol, the subjects went to MEDES for preimmersion muscle biopsy and resting metabolic rate measurement. General protocol of strict DI was conducted according to the methodology detailed in De Abreu et al. [14]. Subjects were paired, one from the Control group and one from the Cuff group. Each pair underwent Dry Immersion simultaneously in the same room, in two separate baths (except for two subjects, one Cuff and one Control, C and M, who had no mate). Thermoneutral water temperature was continuously maintained steady. Light-off period was set at 23:00-07:00. Daily hygiene, weighing and some specific measurements required extraction from the bath. During these out-of-bath periods, subjects maintained the $-6^{\circ}$ head-down position, a reliable position to maintain physiological effects of microgravity used in bedrest studies [15]. Total out-of-bath supine time for the $120 \mathrm{~h}$ of immersion was $9.7 \pm 1.3 \mathrm{~h}$. On DI1-DI4 out-of-bath time was $1.1 \pm 0.6 \mathrm{~h} /$ day. On DI5 out-of-bath time was $5.3 \pm 1.1 \mathrm{~h}$, to carry out muscle biopsy and Magnetic Resonance Imaging (MRI). Otherwise, during DI, subjects remained immersed in a supine position for all activities and were continuously observed by video monitoring. Body weight, blood pressure, heart rate and tympanic body 
temperature were measured daily. The range of adequate water intake was fixed at $35-60 \mathrm{ml} / \mathrm{kg} / \mathrm{day}$; within this range water intake throughout the protocol was ad libitum (measured). The menu composition of each experiment day was identical for all participants and dietary intake was individually tailored and controlled during the study. The individual energy intake was calculated by multiplying resting metabolic rate with physical activity levels of respectively 1.6 and 1.3 before and during DI. Daily caloric intake was around $2625 \mathrm{kcal}$ for baseline and $2160 \mathrm{kcal}$ for the Dry Immersion period. Daily intake for iron was approximately 10 and $15 \mathrm{mg}$. Daily nutrition is detailed in supplementary data (Table S1 and S2). Subjects randomized to Cuffs group wore the thigh cuffs during the 5 days of DI, from $10 \mathrm{~h}$ to $18 \mathrm{~h}$ at DI1 and from $8 \mathrm{~h}$ to $18 \mathrm{~h}$ at DI2-DI5. Thigh cuffs are elastic strips, adapted to each subject to have the same effects on lower limb distensibility as at counterpressure of about $30 \mathrm{mmHg}$. Individual adjustment was determined for each subject with calf plethysmography, performed in the supine position at BDC-2. At DI1, thigh cuffs were put on immediately prior to the onset of immersion at $10 \mathrm{~h}$.

\section{Body composition}

Body mass $(\mathrm{kg})$ was measured on a weighing trolley at baseline and every morning during Dry Immersion. Fat and fat free mass were measured by DEXA (Hologic, QDR 4500 C, MA, USA) 4 days before DI (BDC-4), and after 5 days of DI (DI5). As expected, whole body lean mass decreased significantly Table $2(-2.5 \%, p<0.001)$, as well as leg lean mass $(-2.9 \%, p<0.001)$. The fat mass proportion of the participants remained unchanged (for all $24.0 \pm 2.9 \%$ at BDC-4 vs $23.9 \pm 3.0 \%$ at DI5).

Table 2

Impact of 5 days of Dry Immersion on whole body lean mass and leg (right and left) lean mass. Data are the mean \pm SD. Statistical significance was checked using a two-way ANOVA, sources of variation (time, group and interaction) were tested, and the significance level was set at $0.05(C T L n=9 ;$ CUFF $n=$ 9). $\mathrm{DI}=$ Dry Immersion; $\mathrm{BDC}=$ Baseline Data Collection; $\mathrm{CTL}=$ control group (no thigh cuffs); CUFF = cuffs group (with thigh cuffs); $A L L=$ all subjects. Only time effect is significant with $\# \# \# p<0.001$, no cuffs or interaction effects were observed.

\begin{tabular}{|c|c|c|c|}
\hline & CTL $(n=9)$ & CUFF $(n=9)$ & ALL $(n=18)$ \\
\hline \multicolumn{4}{|c|}{ Whole Body lean mass $(\mathrm{kg})$} \\
\hline BDC-4 & $55.5 \pm 4.7$ & $55.8 \pm 6.7$ & $55.6 \pm 5.6$ \\
\hline DI5 & $54.2 \pm 4.5$ \#\#\# & $54.3 \pm 6.5$ \#\#\# & $54.2 \pm 5.4$ \#\#\# \\
\hline \multicolumn{4}{|c|}{ Leg lean mass (kg) } \\
\hline BDC-4 & $18.3 \pm 2.5$ & $18.4 \pm 1.9$ & $18.3 \pm 2.2$ \\
\hline DI5 & $17.6 \pm 2.1$ \#\#\# & $17.7 \pm 1.7$ \#\#\# & $17.7 \pm 1.9$ \#\#\# \\
\hline
\end{tabular}

\section{Stools collection and metagenomics analysis}


Stool was regularly sampled from subjects without constraints. We included then all subjects with stool sampling before the beginning of DI1 (D0) and during the DI5 (D5). Then we monitored the evolution of gut microbiota composition of each subject before and after the DI $(n=14)$. Subject $E, M, H$, and $R$ were finally excluded as no stool collection was obtained on both days (D0 or D5) hampering statistical paired analysis with these subjects.

DNA extraction from feces. Total cellular DNA was extracted from $0.1 \mathrm{~g}$ of animal fecal material using the G'NOME® kit (BIO 10, MPBio, La Jolla, CA) with modifications [16]. Fecal samples were homogenized in the supplied cell suspension solution. Cell lysis/denaturing solution was then added and samples incubated at $55^{\circ} \mathrm{C}$ for $2 \mathrm{~h}$. To improve cell lysis, $0.1 \mathrm{~mm}$-diameter silica beads $(750 \mu \mathrm{L})$ were added, and samples were mixed at maximum speed in a Fast-Prep (MPBio, La Jolla, CA) for 4 min. Polyvinylpolypyrrolidone (PVPP, $15 \mathrm{mg}$ ) was added to ensure removal of polyphenol contamination that could inhibit the qPCR assays. Samples were vortexed and centrifuged at $20000 \mathrm{~g}$ for $3 \mathrm{~min}$ and supernatants were recovered. The remaining pellets were washed with $400 \mu \mathrm{L}$ of TENP [50 mM Tris (pH 8), $20 \mathrm{mM}$ EDTA (pH 8), $100 \mathrm{mM} \mathrm{NaCl}, 1 \%$ PVPP] and centrifuged at $20000 \mathrm{~g}$ for $3 \mathrm{~min}$. The washing step was repeated once more and the resulting supernatants pooled. Nucleic acids were precipitated by addition of one volume of isopropanol, incubation at $-20^{\circ} \mathrm{C}$ for $20 \mathrm{~min}$ and centrifugation at $20000 \mathrm{~g}$ for $10 \mathrm{~min}$. Pellets were resuspended in $400 \mu \mathrm{L}$ of distilled water plus $100 \mu \mathrm{L}$ of salt-out mixture and incubated at $4{ }^{\circ} \mathrm{C}$ for $10 \mathrm{~min}$. Samples were spun at maximum speed for $10 \mathrm{~min}$, and DNA-containing supernatants transferred to clean $1.5-\mathrm{mL}$ microcentrifuge tubes. DNA was precipitated with two volumes of $100 \%$ ethanol at room temperature for $5 \mathrm{~min}$ followed by centrifugation at $16000 \mathrm{~g}$ for $5 \mathrm{~min}$. DNA was resuspended in $150 \mu \mathrm{L}$ of TE buffer and stored at $-20^{\circ} \mathrm{C}$.

Evaluation of total bacteria by real-time qPCR analysis of bacterial $16 \mathrm{~s}$ rRNA genes. The total bacteria present in the fecal samples of each mouse were evaluated by real-time quantitative PCR (qPCR) targeting "all bacteria" 16S rRNA genes using the universal primers F-bact1369 CGGTGAATACGTTCCCGG and R-prok1492 TACGGCTACCTTGTTACGACTT [16]. Analyses were performed using the StepOne Plus detection system (Applied Biosystems, Courtaboeuf, France) with 10 $\mu \mathrm{L}$ of Mastermix (PowerUpSybrGreen Master Mix, ThermoFisher Scientific, Courtaboeuf, France), $500 \mathrm{nM}$ of both forward and reverse primers, $5 \mu \mathrm{L}$ of diluted cDNA template and water to a final volume of $15 \mu \mathrm{L}$. All qPCR assays were performed in duplicate using the following cycling conditions: $50{ }^{\circ} \mathrm{C}$ for $2 \mathrm{~min}$, then $95^{\circ} \mathrm{C}$ for $2 \mathrm{~min}$ followed by 40 cycles of $95^{\circ} \mathrm{C}$ for $3 \mathrm{~s}$ and $60^{\circ} \mathrm{C}$ for $30 \mathrm{~s}$, with a final melting step to improve the amplification specificity. For the quantification, the Escherichia coli ${ }^{E}$ DNA standard curve was generated by plotting the threshold cycles (Ct) vs. bacterial quantity. When qPCR assays were performed using fecal DNA samples, this standard curve was used to quantify the bacterial population. The lower limit of detection for bacterial enumeration with good precision is $10^{6}$ bacteria per gram of stool.

Phyla abundance quantification by Real-time qPCR. Quantifications of specific phyla were performed using the following probes specific of the 3 main phyla: Firmicutes ${ }^{P}$ (934F-Firm-5'GGAGYATGTGGTTTAATTCGAAGCA-3' and 1060-FirmR-5'-AGCTGACGACAACCATGCAC-3'), Bacteroidetes $^{P}$ (MIBF-5'-GGCGACCGGCGCACGGG-3' and MIBR-5'-GRCCTTCCTCTCAGAACCC-3'), 
ProteobacteriaP $^{P}$ (ProteoF-5'-GCTCGTGTTGTGAAATGTTGG-3' and ProteoR-5'-

CGTAAGGGCCATGATGACTTG-3') with the protocol using for All-bacteria. The total number of bacteria was inferred from averaged standard curves as previously described $[17,18]$. For the quantification of Firmicutes $^{P}$, Bacteroidetes $^{P}$ and Proteobacteria ${ }^{P}$, standard curves were generated from serial dilutions of a known concentration of genomic DNA from Lactobacillus acidophilus ${ }^{E}$, Bacteroides fragilis $^{E}$ and Escherichia coli ${ }^{E}$, respectively.

Evaluation of microbiota composition by sequencing. The V3-V4 region of the 16SrRNA genes was amplified using the bacterial primers 343F (5'-CTT TCC CTA CAC GAC GCT CTT CCG ATC TAC GGR AGG CAG CAG-3') and 784R (5'-GGA GTT CAG ACG TGT GCT CTT CCG ATC TTA CCA GGG TAT CTA ATC CT-3') modified to add adaptors during the second PCR amplification. PCR assays were performed using the MolTaq 16S DNA polymerase and the corresponding master mix (Molzym GmbH \& Co.KG, Bremen, Germany). The PCR mix contained $10 \mathrm{ng}$ of DNA, $1 \mu \mathrm{L}$ of dNTPs $(10 \mathrm{mM}), 1.25 \mu \mathrm{L}$ each of forward and reverse primer $(20 \mu \mathrm{M})$, and $0.5 \mu \mathrm{L}$ of Taq polymerase in a total volume of $50 \mu \mathrm{L}$. The cycling program was: $94{ }^{\circ} \mathrm{C}$ for $3 \mathrm{~min}$, followed by 40 cycles at $94^{\circ} \mathrm{C}$ for $15 \mathrm{~s}, 60^{\circ} \mathrm{C}$ for $30 \mathrm{~s}, 72{ }^{\circ} \mathrm{C}$ for $60 \mathrm{~s}$, and a final extension at $72{ }^{\circ} \mathrm{C}$ or $5 \mathrm{~min}$. Sequencing was performed using the MiSeq technology (Illumina) at the Genopole Toulouse Midi-Pyrenees (GeT) genomics facility (http://get.genotoul.fr/).

Metagenomics analysis. Sequencing data were demultiplexed at the GeT platform. The Galaxy-supported pipeline, called FROGS (Find, Rapidly, Otus with Galaxy Solution), is designed to analyze large sets of amplicon sequences and produce abundance tables of Operational Taxonomic Units (OTUs) and their taxonomic affiliation [19]. The most abundant sequences of each OTUs were then affiliated with blastn against the Silva v128 database [20]. Abundance tables and taxonomy files were manually imported into RStudio (v1.2.1335). To performed analysis package Phyloseq 1.28.0 [21], ggplot2 [22] and custom scripts were used. Samples were rarefied to even sampling depths before computing within-samples compositional diversities (Observed richness, Chao1, Shannon and InvSimpson) and between-samples compositional diversity (Jaccard, Bray-Curtis, UniFrac). Principal Coordinate Analysis (PCoA) were also performed on dissimilarities matrices to obtain a 2-dimensional representation of the samples. Alpha diversity data were analyzed using paired $t$-test when the normality and/or equal variance tests pasted or Wilcoxon rank sum test was chosen. Permutational multivariate ANOVA (PERMANOVA) tests were performed on Jaccard, Bray-Curtis, UniFrac matrices using 9999 random permutations and at a significance level of 0.01 . Phyla relative abundances were compared using paired $t$-test on GraphPad Prism 6.02.

\section{Participant Flow and statistics}

The Fig. 1 represented the participant flow. All data are presented as the mean \pm SEM. The normality of each distribution and homogeneity of variance were assessed with the Kolmogorov-Smirnov and Fischer test respectively. This study was originally designed to assess the effects of thigh cuffs on the hematological, cardiovascular and musculoskeletal system responses induced by 5 days of DI. Statistical significance was checked using a two-way ANOVA for repeated measures. As cuffs did not have any 
significant effects on the gut microbiota composition (Figure S1 and S2) during simulated microgravity, the results and discussion section only focus on the effects of DI on gut microbiota metabolism. The paired $t$-test was used to compare directly D0 vs. D5. The Wilcoxon rank sum test was chosen when the normality and/or equal variance tests failed. For all statistical analyses, the significance level was set at 0.05. Data were analyzed using the statistical package GraphPad Prism version 6.02 for Windows (GraphPad Software, La Jolla, California).

\section{Results}

\section{The abundance of the main phyla is preserved during 5-days of Dry immersion.}

To confirm the equal success and quality of 16S rRNA genes extraction between groups, we performed all bacteria qPCR (Fig. 2a). As expected, extraction quantity is similar between subjects at D0 (12.71 \pm 0.26 In of bacterial cells/g feces) and D5 (12.69 \pm 0.22 In of bacterial cells/g feces), which confirmed the robustness of this method. Our results from qPCR analysis suggest a preserved abundance of the main phyla: Bacteroidetes $^{P}($ D0: $11.66 \pm 0.10 \log 10 ;$ D5: $11.62 \pm 0.08 \log 10)$, Firmicutes ${ }^{P}($ D0: $11.72 \pm 010$ $\log 10 ;$ D5: $11.68 \pm 0.09 \log 10)$ and Proteobacteria ${ }^{P}$ (D0: $9.62 \pm 0.18 \log 10 ;$ D5: $\left.9.67 \pm 0.14 \log 10\right)$. Total quantities of each phyla are similar between times (Fig. 2b). The robustness of all-bacteria quantification allowed us to use it as a control baseline for phyla quantification by qPCR ("housedkeeping" all-bacteria). Statistical analysis of phyla quantification / all bacteria abundance expressed as $2^{-\Delta \Delta C t}$ shows no differences before and after DI (Fig. 2c). This is confirmed by metagenomics analysis reporting no variation in the abundance neither in these 3 main phyla nor in Actinobacteria abundance (see subsequent result paragraph).

\section{5-days of Dry Immersion do not significantly affect $\alpha$ and $\beta$ diversity indices.}

To measure overall changes in men microbiome submitted to $\mathrm{DI}$, we performed $\mathrm{a}$ and $\beta$ diversity analysis of taxonomic profiles for each of the 14 men before and after DI. The following figures illustrate gut microbiota composition before (D0) and after 5-days (D5) of Dry Immersion with 16S rRNA genes metagenomics analysis. Our results on a-diversity do not show significant differences of DI along time on Observed, Chao1, Shannon and InvSimpson indices (Fig. 3a). Moreover, individual plots show similar evolution between subjects in all a-diversity indices (Fig. 3b), indeed no significant differences are observed: Observed $p=0.166$; Chao1 $p=0.984$; Shannon $p=0.121$; InvSimpson $p=0.348$. These findings indicate absence of DI effects on gut microbiota richness and evenness. PCoA plots (Fig. 3c) established from dissimilarity matrices to explain $\beta$-diversity through Jaccard $(p=0.998)$, Bray-Curtis $(p=0.997)$ and UniFrac $(p=0.999)$ indices show any differences between time. These results reveal the absence of DI effect on OTUs absence / presence, abundance and phylogeny differences before (D0) and after (D5) Dry Immersion.

5-days of Dry Immersion increase Lachnospiraceae ${ }^{\mathrm{F}}$ abundance. 
Metagenomic analysis reveals similar results as qPCR analysis for individual and global phyla abundances (Fig. 4a). 5 days of Dry Immersion do not modify 4 main phyla abundances (Fig. 4b). Total abundances of OTUs associated to orders belonging to 4 main phyla are represented in Figure S3. The metagenomics analysis of the 32 families and 44 associated genera showed some significant and interesting results. Indeed, 5 -days of $\mathrm{DI}$ increased OTUs related to $\mathrm{Clostridiales}^{\mathrm{O}}$ order from $65.5 \%$ up to $69.3 \%$ ( $p=0.015 ;$ Fig. $4 c$ ). The 2 others orders Lactobacillaceae ${ }^{O}$ and Selenomonadales ${ }^{0}$ OTUs which represented each $2 \%$ of the Firmicutes $^{P}$ phylum are decreased by Dry Immersion (data not shown). Moreover, Lachnospiracea $e^{F}$ family $\left(\right.$ Firmicutes $\left.^{p}\right)$ relative abundance is increased by DI with a mean value at D0 of $0.202 \pm 0.01$ and a D5 of $0.241 \pm 0.01$ ( $<<0.01$; Fig. 4d).

\section{Discussion}

This work shows for the first time, in healthy men, the effect of 5-days Dry Immersion, an innovative approach that accurately reproduces the effects of microgravity, on gut microbiota composition. $a$ and $\beta$-diversities remained unchanged after DI which is soothing for current and future spaceflights. Indeed, 5 days of a drastic life conditions were not able to modify significantly the diversity neither the phyla abundances, which confirmed the stability of gut microflora. However, it is sufficient to lead to changes at the lower taxonomy levels, notably in OTUs attributable to Clostridiales $^{O}$ order and Lachnospiraceae $^{F}$ family. This sheds new light on the emerged microbiota - microgravity research field.

Our findings on unaltered $\mathrm{a}$ and $\beta$-diversities are partially consistent with the literature. Voorhies and colleagues' study on astronaut's project (AM) which investigated the impact in human of 6 months to 1 year spaceflight did not find any changes in a-diversity before and after the mission. However, they showed a variation of $\beta$-diversity during spaceflight, whereas after the mission they did not find again difference with pre-flight value. Ritchie and colleagues also did not find any change in a-diversity after 13 days of spaceflight in murine model, yet they identified a cluster on $\beta$-diversity between ground and flight groups. To explain $\beta$-diversity stability compared to other studies, we should consider the large variability between humans' gut microbiome composition, small number of subjects and differences with murine models.

It is also not surprising that Dry Immersion did not induce significant global composition changes at phyla levels as already reported in literature on human or murine spaceflight $[10,23]$. Indeed, the duration of DI was limited to 5-days, with nevertheless significant limited effects on the lean mass $(-2.5 \%$ reduction). It is interesting to noteworthy that at the lower taxonomy levels, this ground model of microgravity induced significant alteration in OTUs attributable to Clostridiales $^{\circ}$ order and Lachnospiraceae ${ }^{F}$ family. Dry Immersion increases Clostridiales $^{O}$ OTUs which represent around $70 \%$ of Firmicutes ${ }^{\mathrm{P}}$ phylum and decreased Lactobacillales $^{\mathrm{O}}$, lower expressed order (data not shown). These findings concur with the findings of the Space shuttle Atlantis mission realized on rats during 13 days [23]. Indeed, in this last study, OTUs associated to Clostridiales $^{\circ}$ are increased (+60\%) and Lactobacillales ${ }^{O}$ are decreased (-62\%) after spaceflight. 
The Lachnospiraceae ${ }^{F}$ family is a phylogenetically and morphologically heterogeneous taxon belonging to the clostridial cluster XIVa of the phylum Firmicutes ${ }^{P}$ [24]. This family of anaerobic bacteria impacts their hosts by producing short-chain fatty acids, converting primary to secondary bile acids and facilitating colonization resistance against intestinal pathogens. In our present study, the significant higher abundance of associated OTUs could be interpreted as a positive adaptation to simulated microgravity as its reduction in other studies has been associated with negative health implications. Moreover, its introduction as probiotics succeeded to increase immune resistance against pathogen bacteria exposition as Clostridium difficile ${ }^{E}$ [25]. Nevertheless, Sorbara et al. pointed out in a recent research the inter and the intra-diversity of these commensal bacterial species belonging to Lachnospiraceae ${ }^{F}$, with, for example, Ruminococcus Gnavus ${ }^{E}$ incriminated in Crohn's disease pathogenesis [26]. These results highlight the potential impact of microbiota changes on systemic inflammation and immune component of microgravity [1]. Interestingly, increase of Lachnospiraceae ${ }^{F}$ OTUs is also reported in hindlimb mice model use to simulate microgravity [27] and divers genera and species belonging to Lachnospiraceae ${ }^{F}$ are also increased after 37 days in ISS [28]. Even in hypergravity mice model Lachnospiracea ${ }^{F}$ is increased [29]. Lachnospiraceae ${ }^{F}$ family seems to play a central role in gravity - gut microbiota axis which calls for further researches.

Collectively, our findings offer new valuable data and information suggesting that human gut microbiota is sensitive to microgravity justifying the need to investigate further this new field of research. More studies, involving a larger number of individuals are now needed to evaluate and identify the impact of microgravity on gut microbiota using recognized, adapted models and targeted countermeasures. Our results on gut microbiome during DI joining those obtained from spaceflights reinforce and justify DI model in this emerging problematic. The head-down bed rest which has historically been the model for exploring the effects of weightlessness and hypoactivity in humans can be used on longer periods, ranging from a few days to several months, and resulting in a considerable reduction in the activity of the different muscle groups, (especially postural) in proportions close to that which is observed in astronauts [15]. In the context of the regulation of human gut microbiota and long - time spaceflights, the use of long - time bedrest model would be thus brought helpful and complementary data to DI but currently, no studies are reported. Indeed, it is the full integration of all the data that will lead to propose recommendations for microbiota - based human efficient countermeasures for astronauts.

\section{Conclusion}

Our study is the first one to analyze the impact of Dry Immersion on human gut microbiota. We showed that the diversity and global composition of the gut microbiome are preserved during 5 days of DI. However, this short-term protocol is sufficient to lead to several significant changes at the lower taxonomy levels that may be related to the induced - immune response. Our study produced new valuable data which are consistent with those obtained from other spaceflight studies. This confirms that gut bacteria are sensitive to simulated microgravity and necessitates further research in order to identify bacteria clusters regulated by Dry Immersion, bed-rest and spaceflights. Finally, based on our results 
collected, and beyond the countermeasure proposed (artificial gravity, exercise...), the aim would be to pave the way for the use of specific new probiotics, as innovative nutritional countermeasures to optimize astronaut's health and performance, in particular in the context of long duration spaceflights.

\section{Abbreviations}

AM

Astronaut's Microbiome

AMPK

AMP-Activated protein kinase

ANSM

French National Agency for Medicines and Health Products Safety

BDC

Baseline Data Collection

CPP

Comité de Protection des Personnes

Ct

Threshold Cycles

CTL

Control group

DI

dry immersion

dNTP

Deoxynucleotide Triphosphate

DPB

Diastolic Blood Pressure

FLASH

Fast Length Adjustement of Short reads

FoxO3

Forkhead box 03

FROGS

Finds, Rapidly, Otus with Galaxy Solution

GeT

Genopole Toulouse Midi-Pyrénées

GF

Germ Free

$\mathrm{HR}$

Heart Rate

ISS

International Space Station 
MRI

Magnetic Resonance Imaging

NASA

National Aeronautics and Space Administration

OTUs

Operational Taxonomic Units

PCoA

Principal Coordinates Analysis

$\mathrm{R}$

Recovery

SPB

Systolic Blood Pressure

WBC

White Blood Cells

\section{Declarations}

\section{Ethics approval and consent to participate}

The experimental protocol conformed to the standards set by the Declaration of Helsinki and was approved by the local Ethic Committee (CPP Est III: October 2, 2018, n ID RCB 2018-A01470-55) and French Health Authorities (ANSM: August 13, 2018, ClinicalTrials.gov Identifier: NCT03915457). All participants provided informed consent. The trial is registered in the ClinicalTrialsGov (NCT03915457).

\section{Consent for publication}

All authors have read and approved the paper for submission.

\section{Availability of data and materials}

The datasets used and/or analysed during the current study are available from the corresponding author on reasonable request.

\section{Competing interests}

The authors declare that they have no competing interests. 


\section{Fundings}

This work was supported by the Centre National d'Etudes Spatiales who directed the design and the collection of clinical data. The work was also partially supported by an AlimH department grant for analysis. Université de Montpellier, INRAE and Brittany Council covered the cost of wages and equipment.

\section{Author's contributions}

MJ, BG, GG-K, FD and CK-R, designed the clinical study.

$B G, M J, B V, K N, M-P B, A B e$, and CK-R performed the experiments;

MJ, BG, MM, OR, ABo, FD and CK-R analyzed the data;

MJ, BG, MM, OR, VO, ABo, FD and CK-R interpreted the results of the experiments;

MJ, BG, and CK-R prepared the figures;

MJ and CK-R drafted the manuscript;

$M J, B G, A B o, V O, F D$ and $C K-R$ edited and revised the manuscript;

$M J, B G, M M, V O, B V, A B o, K N, M-P B, A B e, G G-K, F D$ and $C K-R$ read and approved the final manuscript.

\section{Acknowledgements}

The authors thank the volunteers and the staff of MEDES for their participation in this protocol at the MEDES space clinic in 2018 and in 2019. Special thanks are due to Corinne Lombard, coordinator in charge of sampling and Pascale Vasseur in charge of the nutrition follow-up. This work was supported by the Centre National d'Etudes Spatiales. The work was also supported by INRAE and Brittany Council and partially supported by an AlimH department grant. The authors wish to thank Veronique Douard for her gift of phyla primers and George Dambassis and Anthony Giannoulis for their expert manuscript editing.

\section{References}

1. Crucian BE, et al. Immune System Dysregulation During Spaceflight: Potential Countermeasures for Deep Space Exploration Missions. Front Immunol. 2018;9:1437. 
2. Clemente $\mathrm{JC}$, et al. The impact of the gut microbiota on human health: an integrative view. Cell. 2012;148(6):1258-70.

3. van de Guchte M, Blottiere HM, Dore J. Humans as holobionts: implications for prevention and therapy. Microbiome. 2018;6(1):81.

4. Hooper LV, Macpherson AJ. Immune adaptations that maintain homeostasis with the intestinal microbiota. Nat Rev Immunol. 2010;10(3):159-69.

5. Harmsen HJ, de Goffau MC. The Human Gut Microbiota. Adv Exp Med Biol. 2016;902:95-108.

6. Nay K, et al. Gut bacteria are critical for optimal muscle function: a potential link with glucose homeostasis. Am J Physiol Endocrinol Metab. 2019;317(1):E158-71.

7. Lahiri S, et al., The gut microbiota influences skeletal muscle mass and function in mice. Sci Transl Med, 2019. 11(502).

8. Mohr AE, et al. The athletic gut microbiota. J Int Soc Sports Nutr. 2020;17(1):24.

9. Garrett-Bakelman FE, et al., The NASA Twins Study: A multidimensional analysis of a year-long human spaceflight. Science, 2019. 364(6436).

10. Voorhies AA, et al. Study of the impact of long-duration space missions at the International Space Station on the astronaut microbiome. Sci Rep. 2019;9(1):9911.

11. Shul'zhenko EB, et al., [The antigravity suit, chamberless type, as a means of increasing orthostatic tolerance after water immersion hypokinesis and acceleration]. Kosm Biol Aviakosm Med, 1983. 17(6): p. $30-3$.

12. Tomilovskaya E, et al. Dry Immersion as a Ground-Based Model of Microgravity Physiological Effects. Front Physiol. 2019;10:284.

13. Navasiolava NM, et al. Long-term dry immersion: review and prospects. Eur J Appl Physiol. 2011;111(7):1235-60.

14. De Abreu S, et al. Multi-System Deconditioning in 3-Day Dry Immersion without Daily Raise. Front Physiol. 2017;8:799.

15. Traon P-L. A., et al., From space to Earth: advances in human physiology from 20 years of bed rest studies (1986-2006). Eur J Appl Physiol. 2007;101(2):143-94.

16. Mariat $D$, et al. The Firmicutes/Bacteroidetes ratio of the human microbiota changes with age. BMC Microbiol. 2009;9:123.

17. Nakanishi $Y$, et al. Increase in terminal restriction fragments of Bacteroidetes-derived 16S rRNA genes after administration of short-chain fructooligosaccharides. Appl Environ Microbiol. 2006;72(9):6271-6.

18. Furet JP, et al. Comparative assessment of human and farm animal faecal microbiota using realtime quantitative PCR. FEMS Microbiol Ecol. 2009;68(3):351-62.

19. Escudie F, et al. FROGS: Find, Rapidly, OTUs with Galaxy Solution. Bioinformatics. 2018;34(8):128794. 
20. Quast C, et al. The SILVA ribosomal RNA gene database project: improved data processing and webbased tools. Nucleic Acids Res. 2013;41(Database issue):D590-6.

21. McMurdie PJ, Holmes S. phyloseq: an R package for reproducible interactive analysis and graphics of microbiome census data. PLoS One. 2013;8(4):e61217.

22. Wickham H, ggplot2: Elegants Graphics For Data Analysis. Springer Book Archive. 2009.

23. Ritchie LE, et al. Space Environmental Factor Impacts upon Murine Colon Microbiota and Mucosal Homeostasis. PLoS One. 2015;10(6):e0125792.

24. Rainey FA. The Firmicutes. In: Whitman, editors: Bergey's Manual of Systematic Bacteriology. Heidelberg., Editor.: D. Springer; 2009. p. 921 in P. De Vos, G.M. Garrity, D. Jones, N.R. Krieg, W. Ludwig, F.A. Rainey, K. Schleifer, and W.B.

25. Buffie CG, et al. Precision microbiome reconstitution restores bile acid mediated resistance to Clostridium difficile. Nature. 2015;517(7533):205-8.

26. Sorbara MT, et al. Functional and Genomic Variation between Human-Derived Isolates of Lachnospiraceae Reveals Inter- and Intra-Species Diversity. Cell Host Microbe. 2020;28(1):134-46 e4.

27. Shi J, et al. Intestinal microbiota contributes to colonic epithelial changes in simulated microgravity mouse model. FASEB J. 2017;31(8):3695-709.

28. Jiang $\mathrm{P}$, et al. Reproducible changes in the gut microbiome suggest a shift in microbial and host metabolism during spaceflight. Microbiome. 2019;7(1):113.

29. Alauzet C, et al. Hypergravity disrupts murine intestinal microbiota. Sci Rep. 2019;9(1):9410.

30. Ren D, et al. Mechanism of cholesterol reduction to coprostanol by Eubacterium coprostanoligenes ATCC 51222. Steroids. 1996;61(1):33-40.

31. Li L, et al. Effect of feeding of a cholesterol-reducing bacterium, Eubacterium coprostanoligenes, to germ-free mice. Lab Anim Sci. 1998;48(3):253-5.

32. Larsen JM. The immune response to Prevotella bacteria in chronic inflammatory disease. Immunology. 2017;151(4):363-74.

\section{Description Of Supplementary Files}

Supplemental Table S1. Overall daily nutrition during the study protocol.

Supplemental Table S2. Vitamin intakes during the study protocol

Supplemental Fig $\mathbf{S 1}$. Absence of thigh-cuffs effect on a-diversity gut microbiota before (D0) and after (D5) 5-days Dry Immersion in healthy men ( $n=7 /$ group).

Supplemental Fig S2. Absence of thigh-cuffs effect on $\beta$-diversity gut microbiota before (D0) and after (D5) 5-days Dry Immersion in healthy men ( $n=7 /$ group). 
Supplemental Fig S3. Graphical representations of OTUs affiliated to families belonging to Actinobacteria $^{P}$, Bacteroidetes $^{P}$, Firmicutes $^{P}$ and Proteobacteria $^{P}$ before (D0) and after (D5) 5-days Dry Immersion in healthy men ( $n=14$ /group).

\section{Figures}

Fig. 1

\section{Participant flow chart diagram}

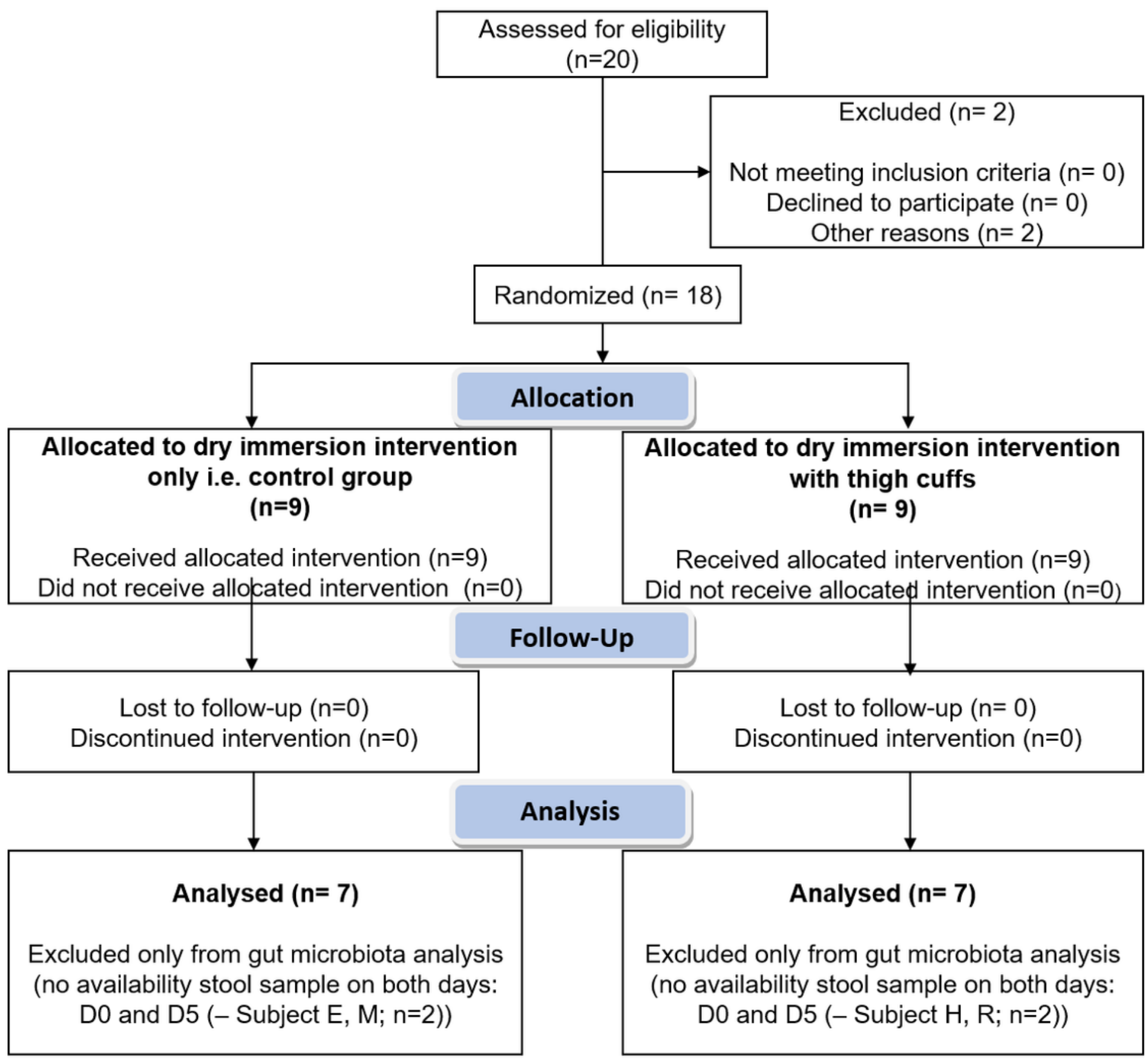


Participant flow chart diagram. 20 subjects were recruited to participate to Dry Immersion experimentation during 5 days. Before randomization, 2 subjects were excluded for other reasons than a mismatch with inclusion criteria or decline to participate. 8 subjects were divided in 2 groups: Control group ( $n=9$ ) and CUFF group (with thigh cuffs; $n=9$ ) and followed-up 5 days of Dry Immersion. Tools were sampled at D0 and D5 and then bacterial abundance were analyzed by qPCR and metagenomic followed-up by bio-statistics. D0 = before Dry Immersion; D5 = after Dry Immersion.

Fig. 2

a

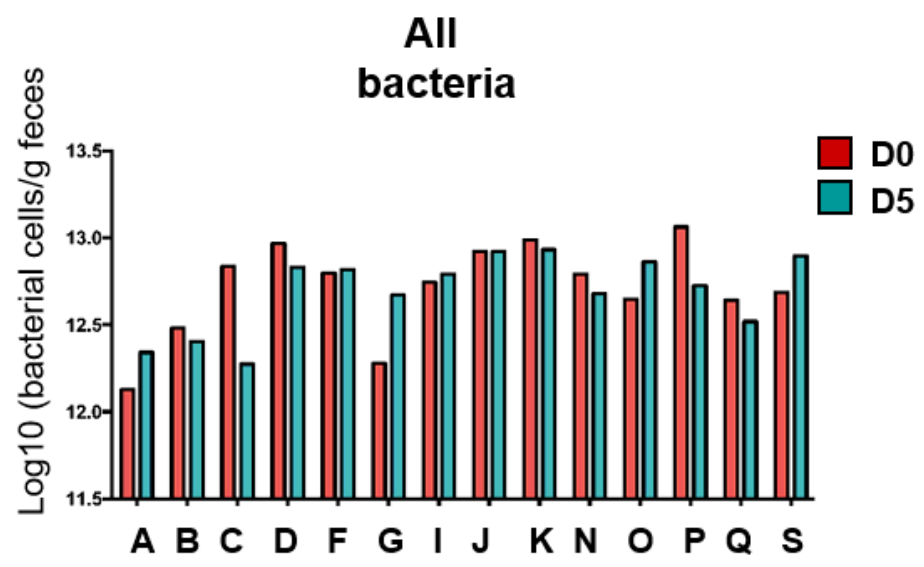

b

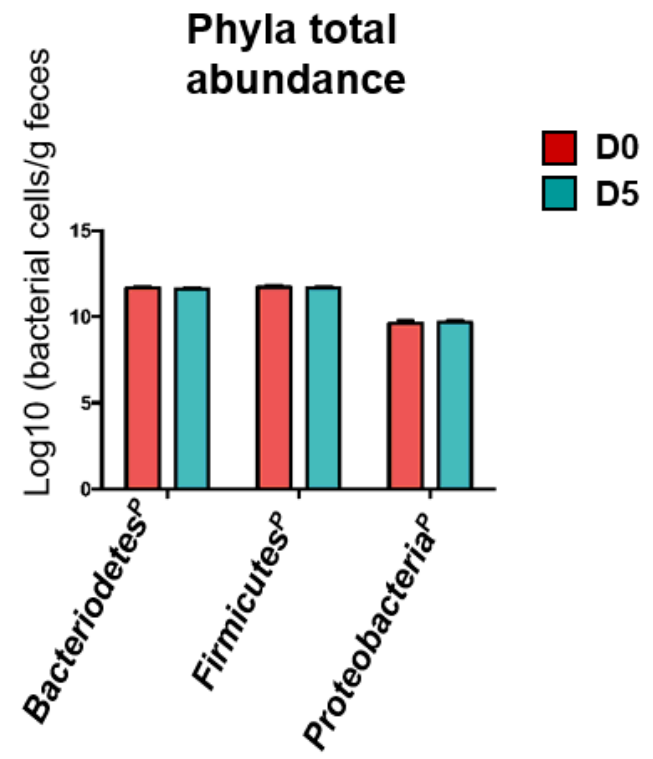

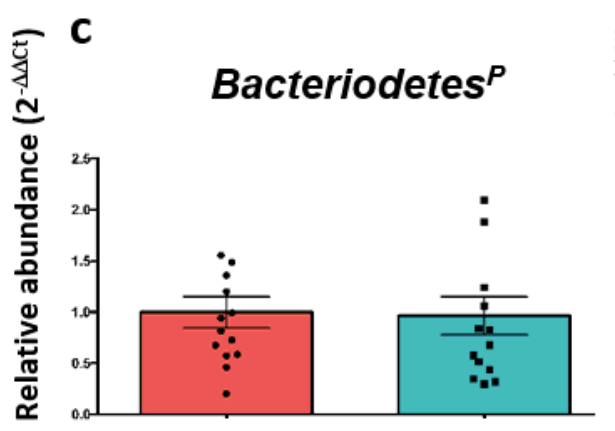

Do

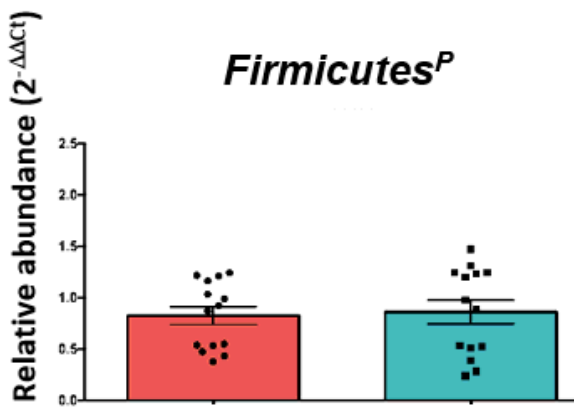

D0

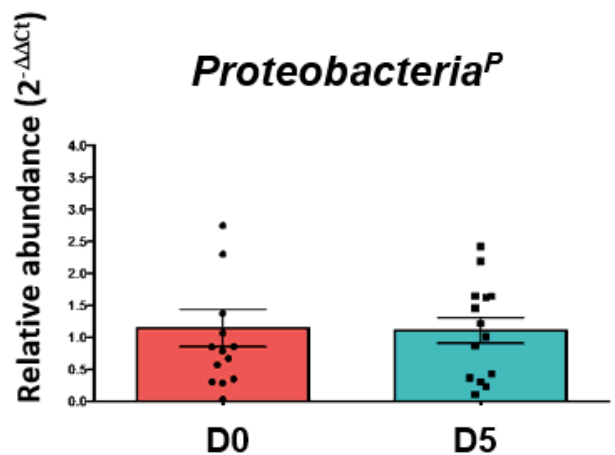

\section{Figure 2}

Gut microbiota composition analysis by qPCR. a All bacteria abundance evaluates by qPCR before (D0) and after (D5) 5-day Dry Immersion in healthy men $(n=14)$. $Y$ axis as subject. Paired t-test performed on GraphPad Prism without significant differences. b Mean abundance of phyla quantifying by qPCR before D0 and after D5 (5-day Dry Immersion) in healthy men ( $n=14)$. Paired t-test performed on GraphPad Prism without significant differences. c Abundance of each phylum by QPCR normalized by all bacteria abundance before (D0) and after (D5) 5-day of Dry immersion in healthy men ( $n=14)$. Paired t-test 
performed on GraphPad Prism without significant differences. D0: before Dry Immersion; D5: after Dry Immersion; $\mathrm{P}=$ Phylum.

Fig. 3

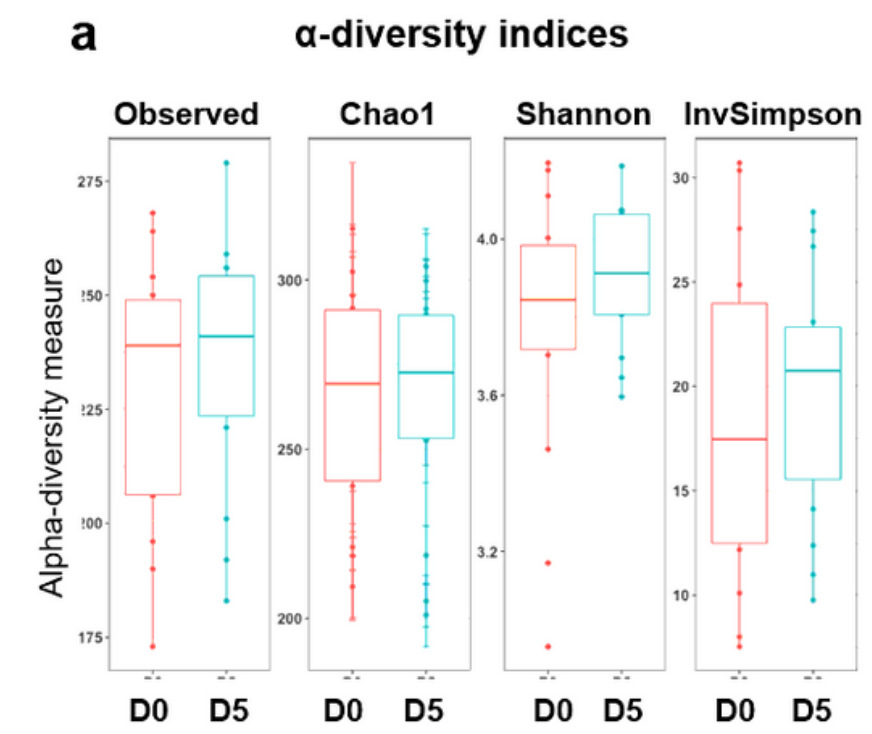

\section{b Individual subject $\alpha$-diversity}
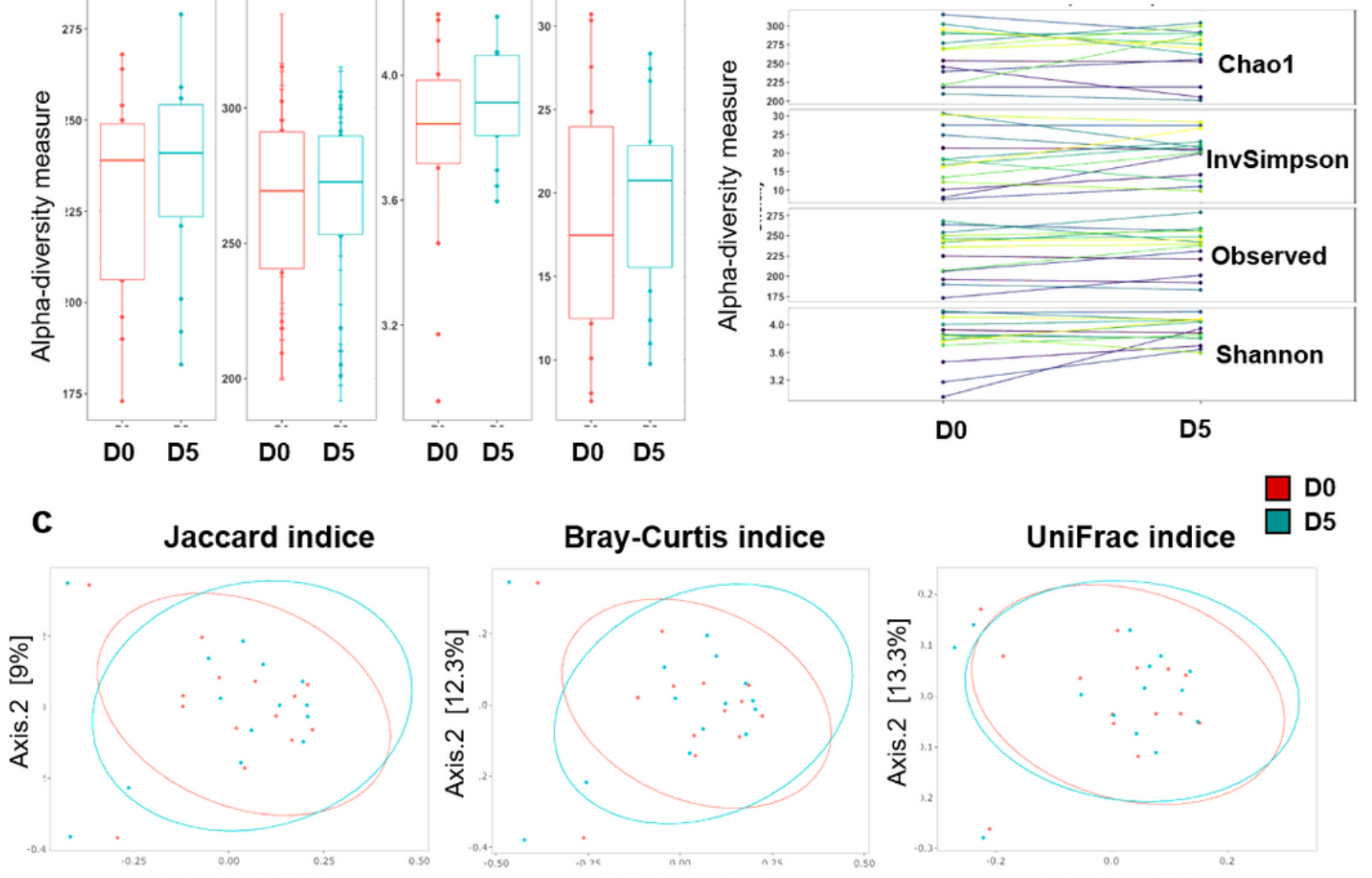

Axis.1 [12.1\%]
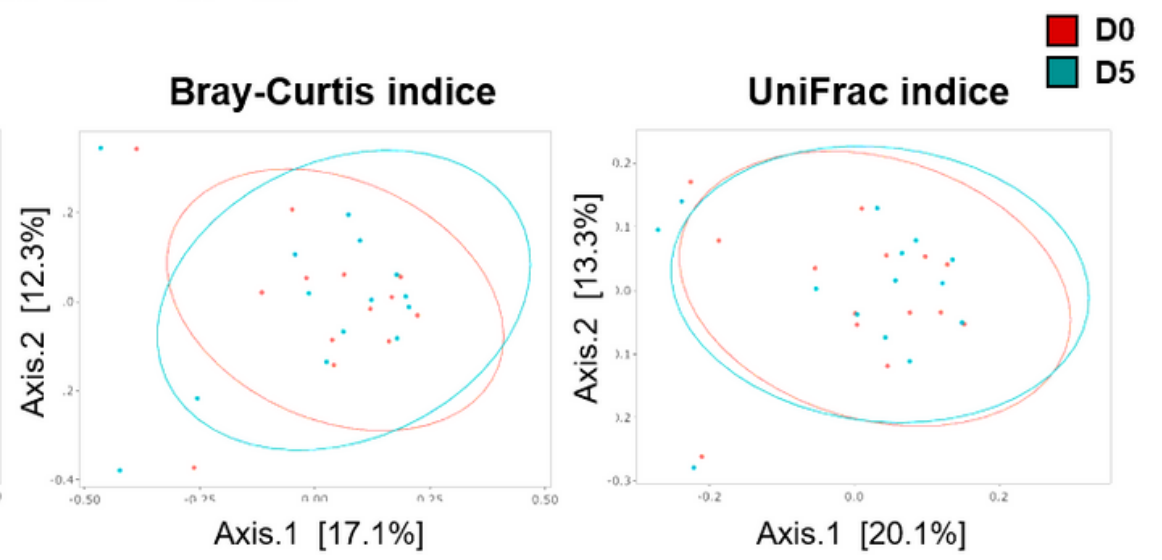

\section{Figure 3}

$a$ and $\beta$-diversity markers before (D0) and after (D5) 5-day Dry Immersion in healthy men ( $n=14)$. a a diversity evaluates with Observed, Chao1, Shannon and InvSimpson indices. Paired t-test performed on GraphPad Prism without significant differences. b Individual a-diversity evaluates with Observed, Chao1, Shannon and InvSimpson indices. $c \beta$-diversity analysis by Jaccard $(p=0.998)$, Bray-Curtis $(p=0.997)$ and UniFrac $(p=0.999)$ indices indicated no difference in microbial OTUs absence / presence, abundances or phylogeny. Statistical PERMANOVA Analysis were performed on RStudio. D0: before Dry Immersion; D5: after Dry Immersion. 
Fig. 4

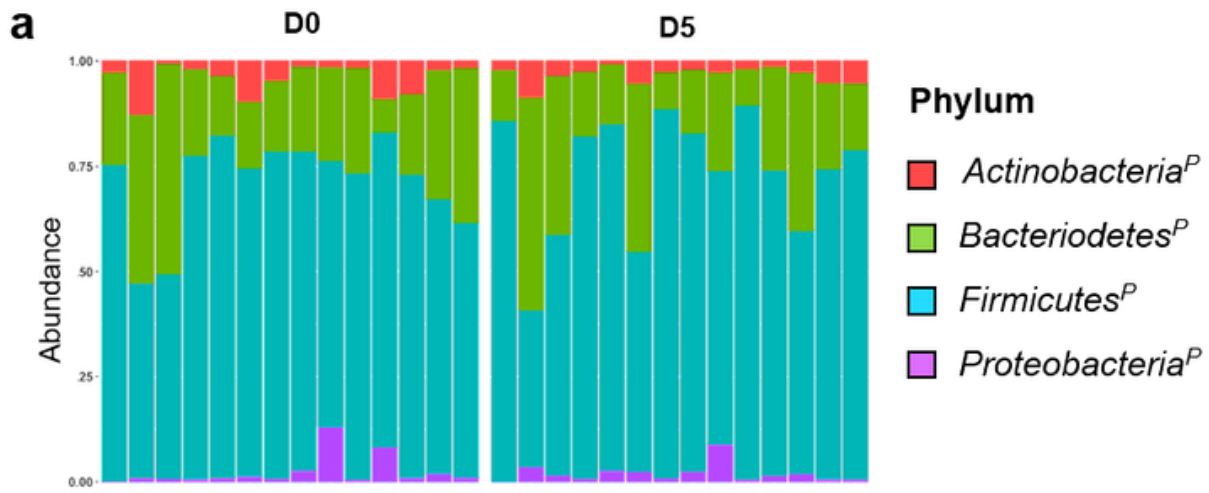

A BCDFG I JKNOPQS A BCDFG I JK NOPQS

b
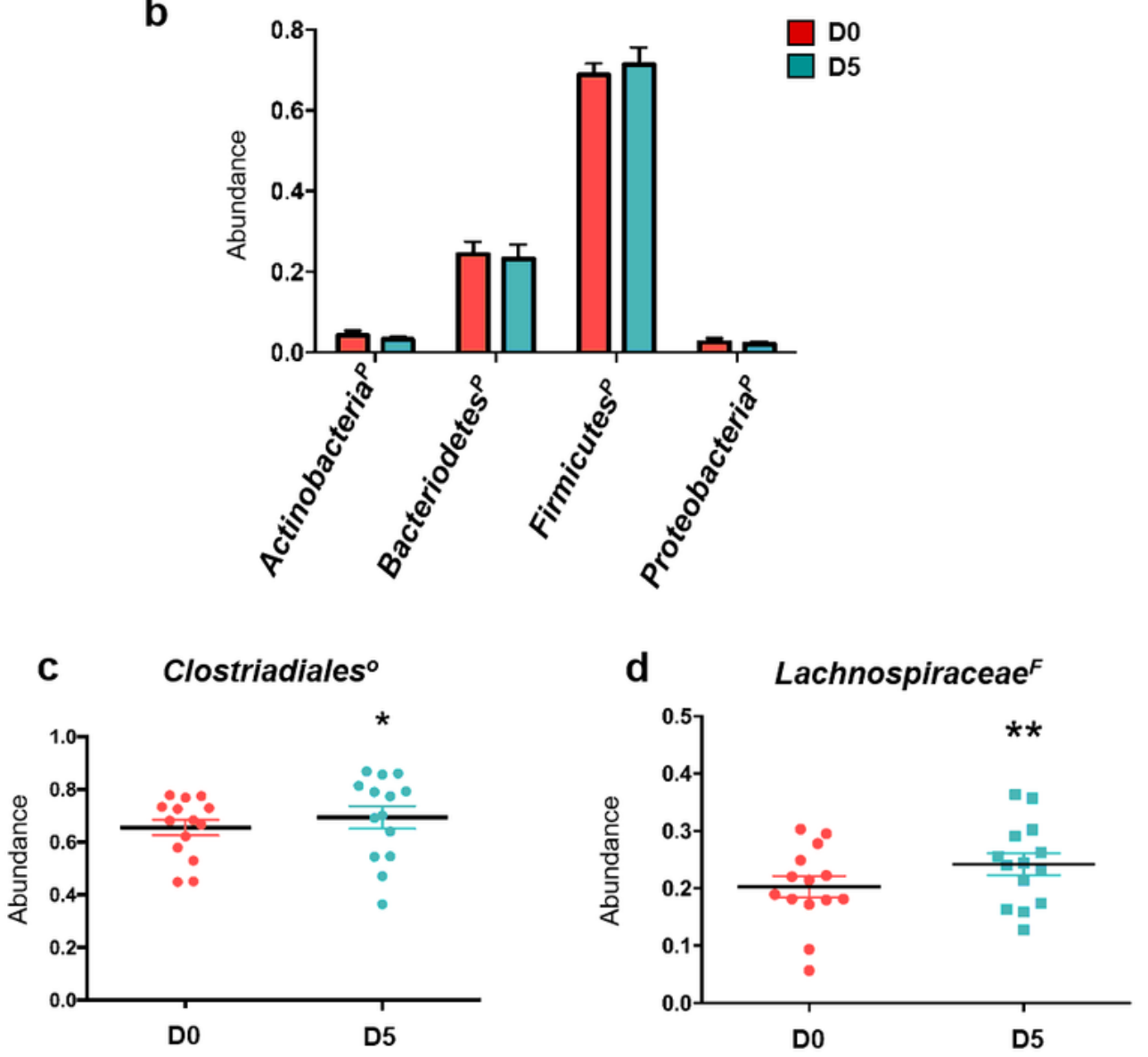

Figure 4

Abundance of phyla obtained by 16S rRNA gene sequencing a Individual abundance of 4 human main phyla before (D0) and after (D5) 5-day Dry Immersion in healthy men ( $=14)$. b Statistical analysis of phyla abundance before (D0) and after (D5) 5-day Dry Immersion in healthy men ( $n=14)$. t-test were performed for each phylum. c ClostridalesO order abundance is significantly increased by DI. Analyze by paired t-test. $d$ LachnospiraceaeF family abundance is heightened by 5 day of DI. Analyse by paired t-test. 
DI = Dry Immersion; D0: before Dry Immersion; D5: after Dry Immersion; $\mathrm{p}=$ Phylum; O = Order; F = Family; $*<p<0.05 ; * \star p<0.01$.

\section{Supplementary Files}

This is a list of supplementary files associated with this preprint. Click to download.

- Tables1.tif

- Tables2.tif

- Figures2.tif

- FigureS1.tif

- FigureS3.tif 